\title{
FRONTISPIECE
}

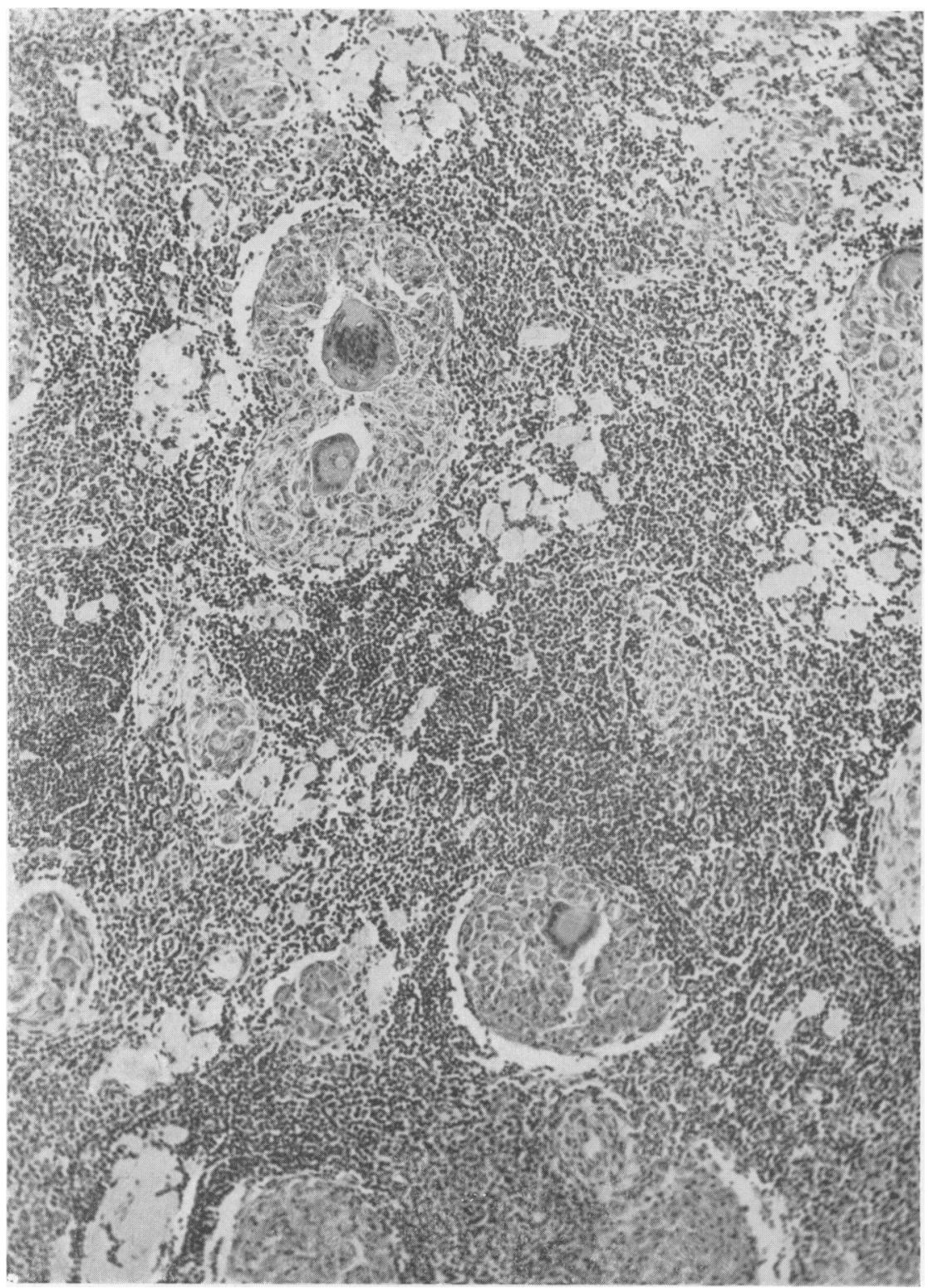

Sarcoid foci in a lymph gland

Illustration to DR. W. M. Macleod's article on Sarcoidosis. Page 217. 


\title{
SARCOIDOSIS
}

\author{
By W. M. Macleod, M.B.E., M.R.C.P. \\ Chest Physician, Southampton
}

Just over 50 years ago a young policeman consulted Professor C. Boeck on account of a troublesome skin disease. The rash was peculiar. It had started on his brow and spread in the course of two years to produce scattered well-defined nodules on his face, scalp and trunk and on the extensor aspects of his limbs. The lesions varied in size from that of a pea to that of a large bean. Most of them were yellowish-brown in colour, though some of the larger ones had a bluish-red tint. In addition to the rash, Boeck noticed that the cubital and femoral groups of glands were greatly enlarged and the axillary group was also easily palpable. Histologically the appearance resembled a sarcoma. This latter observation, together with the indolent clinical progress, led Boeck to call the disease ' multiple benign sarkoid ' (Boeck, 1899).

The picture that Boeck recorded is now known to be but one facet of a disease characterized by disseminated granulomatous lesions which may affect most structures of the body. From his original annotation the term 'sarcoidosis' has developed, and in the English-speaking countries has come to stay as a convenient, though not informative, title for this interesting disease group.

Jonathan Hutchinson ( 1898$)$ is credited with the first report of this condition. In 1878 he reported an anomalous disease of the skin and fingers. Twenty years later he published details of four similar cases. The first concerned a Mrs. Mortimer and he called the condition Mortimer's disease.

Following these earlier papers there have been numerous accounts illustrating various clinical features under a variety of headings and eponyms. The underlying common pathological development was suggested by Schaumann in 1914 (Schaumann, 1924), but the fuller appreciation of the pleomorphic clinical features is more recent. For a more detailed historical account see the papers of Hunter (1936) and Freiman (1948). The latter paper has a most helpful list of references on all aspects of the disease.

Sarcoidosis is a disseminated granuloma of multiple aetiology which, affecting most parts of the body, clinically presents with predominant involvement of the lymph glands, lungs, skin, eyes and bones.

The foci, irrespective of their situation, have a characteristic histological pattern (Ricker and Clark, 1949), though the picture is not specific for this disease (frontispiece). The lesions are composed of large epithelioid cells with abundant acidophilic cytoplasm and large ovoid vesicular nuclei. The cells at times tend to be arranged concentrically. Giant cells are often present and are either of the Langhans or foreign body types. They may show inclusion bodies. There are usually a few lymphocytes scattered at the periphery of the lesion, the whole being bound bP a reticulum. As the lesions grow they fuse, bue their multi-focal development remains recognizo able. In common with the typical clinical features? many authorities will accept variations of the usual pattern. Giant cells may be numerous and lymphocytes moderately increased in number. Those workers who consider sarcoidosis closely related to tuberculosis will even allow minimal caseation as distinct from the more usual central fibrillary necrosis (Rubin and Pinner, 1944).

The foci may resolve, may remain stationary for months or even many years, or may heal, forming hyaline fibrous nodules. The organs involved frequently show enlargement, but it is not rare to find scarcely palpable lymph glands with their normal structure almost entirely replaced by sarcoid tissue.

It has been well recognized for many years that a low skin sensitivity to tuberculin is a frequent occurrence in sarcoidosis. Wells and Wylie (1949) have shown that there is present in the serum of some cases of sarcoidosis a tuberculin-neutralizing factor. While this factor is also found in sera from other diseases, such as kala-azar, and in some apparently normal sera, they believe that its presence in sarcoidosis may be significant. A positive skin reaction to $I$ in 10,000 O.T. might call for a careful review of the evidence, but in the presence of the characteristic clinical picture, especially if backed by histological examination, it should not negative the diagnosis. 
Lemming (1940) was the first of several observers who inoculated patients suffering from sarcoidosis with B.C.G. In general, it was found that conversion to a positive tuberculin reaction was difficult to achieve, and that when it occurred it was of short duration. This response has been used to support the tuberculous aetiology of the disease. Israel and his associates (1950), confirming Lemming's results, point out that these patients also respond unusually to other inoculations such as pertussis vaccine, and that tuberculin anergy is only one feature of a wider interference of their immunologic mechanisms.

Lemming (1942) also noted that the lesion at the site of the B.C.G. inoculation, or in the draining glands, showed a typical sarcoid picture. This histological pattern response is a non-specific one. Kveim (I94I) has claimed that his inoculum derived from a proven sarcoid lymph gland produces a specific skin reaction in this condition. The delayed interval of its appearance and its duration are characteristic. Nelson (1949) has recently discussed this test, and notes that among other substances normal splenic extracts may give similar results: The microscopy of the papules formed shows appearances of sarcoidosis. Nelson's results were not confirmed by Danbolt (I95I), who strongly supports the specificity of Kveim's test.

\section{Clinical Features}

In England, judged by the comparatively small number of series published, it would appear that all sections of the population are equally liable to the disease. In America, on the other hand, there is a much greater incidence among negroes. Both sexes are affected, though it is less common in males. This bias is very evident in the group of cases presenting with erythema nodosum, such cases being nearly always found in women of child-bearing age. The majority of all groups are noted in young and middle-aged adults, but cases have been reported in children and infants, and are not rare in the very old.

One of the most striking features of the disease is the great disproportion between the symptoms and the extent of the lesions. It is well known that a grossly abnormal lung picture often accompanies a reasonably fit and uncomplaining patient who has innocently attended for mass X-ray.

General symptoms are not a prominent feature in the earlier stages of the disease. Loss of weight is slight and notable fever uncommon, though occasionally it may accompany a fairly acute onset of the disease with marked enlargement of the liver and spleen. The E.S.R. is often normal, and a raised total serum globulin with reversal of the albumin-globulin ratio is not an essential feature: when found it is in no way specific to the disease. Other biochemical investigations arez usually normal unless the localization of the disease is such as to affect function, for example inc. the rare involvement of the kidneys.

It is usual for sarcoidosis to present as a local $\stackrel{\overrightarrow{\bar{N}}}{+}$ disease affecting one organ or one particularo structure. The chest physician, the dermatologist

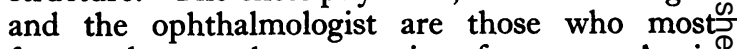
frequently see the presenting features. As is evident from the historical appreciation of the disease so, even today, the clinician is apt to focus his attention on but one aspect of the disease. The danger lies in his treating this fragment $\vec{\omega}$ without due regard for the whole patient and allo the structures affected.

It is simplest to consider the main features in groups.

\section{The Lymph Glands}

It is probably true to say that in every case of sarcoidosis at least one group of glands is diseased. Generalized gross enlargement of the peripheral ${ }_{-}$ glands is not common. When it occurs there is $\vec{\nabla}$ usually enlargement of the liver and spleen ando accompanying symptoms of ill health and fever. This is an uncommon presentation. As a rugec one group of glands is picked out. These magn be superficial, such as the cervical group, but the mediastinal and broncho-pulmonary glands are frequently involved. Occasionally the mediastinalo groups are particularly noticeable, showing $a_{\%}^{\Perp}$ greatly enlarged broad upper mediastinal shadow on the X-ray (Fig. 2). This picture is common $\overrightarrow{\overrightarrow{0}}$ to many lymphadenopathies.

It is more usual, however, for the emphasis to be on the broncho-pulmonary groups. The? resulting gross bilateral hilar enlargement, often with well-marked circular contours in a com-3. paratively well patient is, in England, almostodiagnostic of sarcoidosis (Fig. 3). In any case of suspected sarcoidosis, where confirmatory evidence is required, a careful examination of all peripheral $₹$ groups should be made, as even hardly palpable윽 glands may show the characteristic histology.

\section{The Lungs}

Sometimes a patient showing only broncho- N pulmonary adenopathy will, in the course of a 0 month or two, develop radiological evidence of $\tilde{\omega}$ pulmonary dissemination (Fig. 4). As the lungo foci are chalked in, the hilar shadows are smudgedo out. The glands return to a more normal size, $\mathbb{\complement}$ though still diseased. These changes are not? accompanied by any subjective change in the well-being of the patient, and it is remarkable how $\frac{\mathrm{O}}{\mathbb{D}}$

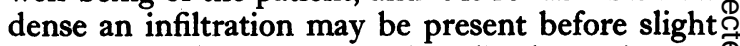
shortness of breath is noted. The lung changes $\stackrel{\mathbb{Q}}{2}$ 


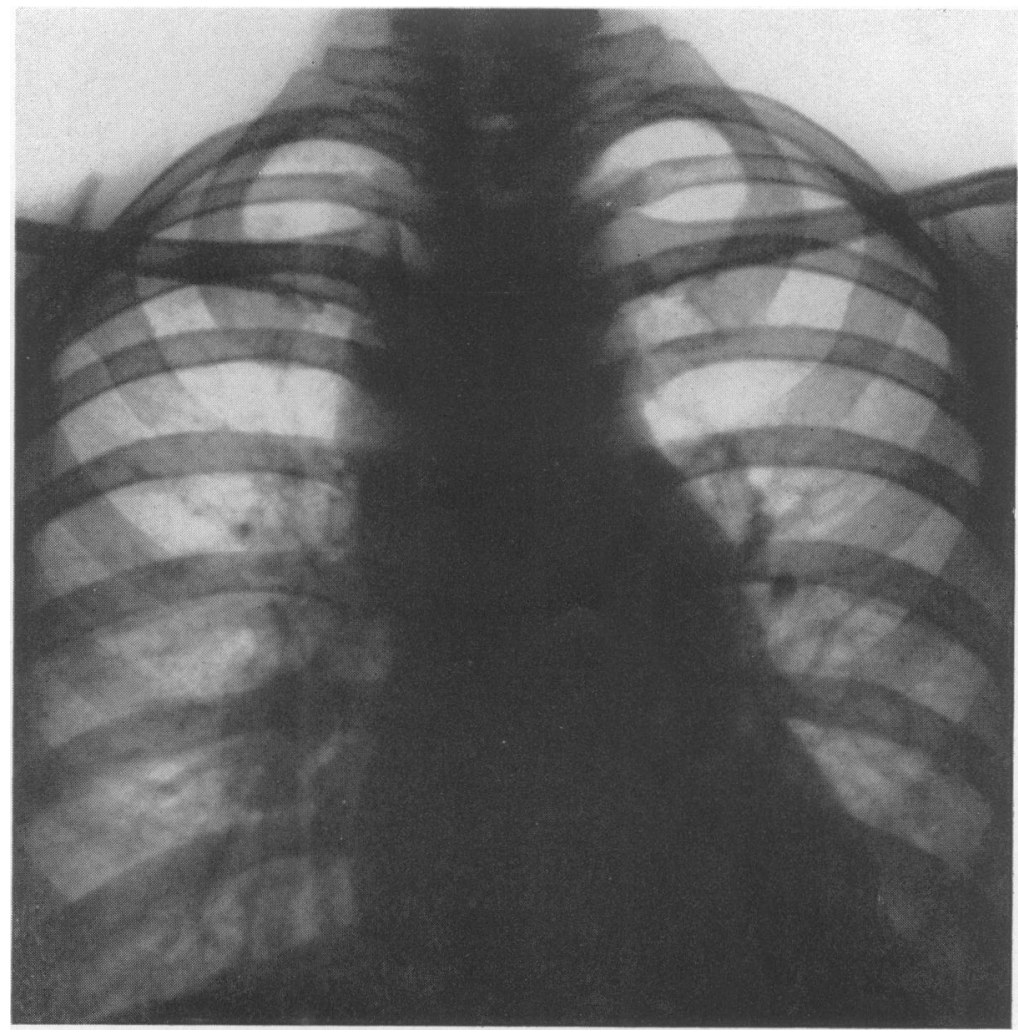

FIG. 2.-X-ray showing wide upper mediastinal shadow. The right hilar shadow is also prominent. There is only slight mottling in the lung fields.

may develop while the glands remain prominent, or they may be present without any known preceding adenopathy. Their main characteristic is that of multiple scattered foci without, as a rule, any major areas of confluence. The very fine mottling seen in some cases of miliary tuberculosis is not usual, but all other variations of size, density and distribution may occur. None are diagnostic. The changes, generally bilateral, may be confined to one lung or lobe. Pulmonary tuberculosis may be closely mimicked, though cavitation, if present, is an interesting rarity.

In favourable cases the mottling reaches its zenith after several months and then, remaining unchanged for an interval which may be as long as two or three years, it clears sometimes with remarkable speed over a few weeks, but as a rule as slowly as it had previously progressed.

Persistence of the infiltration is unfortunate as it is a prelude to disseminated pulmonary fibrosis and associated emphysema. These late changes are recognizable by the complaint of increasing breathlessness. Right-sided cardiac failure may ensue. An appreciable number of cases develop phthisis-variously estimated at ro to 25 per cent. Such definite tuberculous change may involve only part of the sarcoid disease, and is usually accompanied by a change from tuberculin anergy to sensitivity. A similar change in tuberculin sensitivity may occur in cases which are progressing favourably.

Direct involvement of the larger bronchi is rare, and their compression by enlarged glands also very uncommon. Both these features are notably different to the behaviour of proven tuberculous infection. Pleural effusions are also most unusual though cases are occasionally seen which present in such a way.

The course of the lung disease is so variable and unpredictable that the prognosis of any particular case is difficult to determine. A rough clinical impression suggests that about half of the cases presenting with lung disease will resolve apparently completely. Dense infiltrations can eventually clear even though they have been present for two or three years. However it is true that the longer the disease is present the more likely will fibrosis develop. 


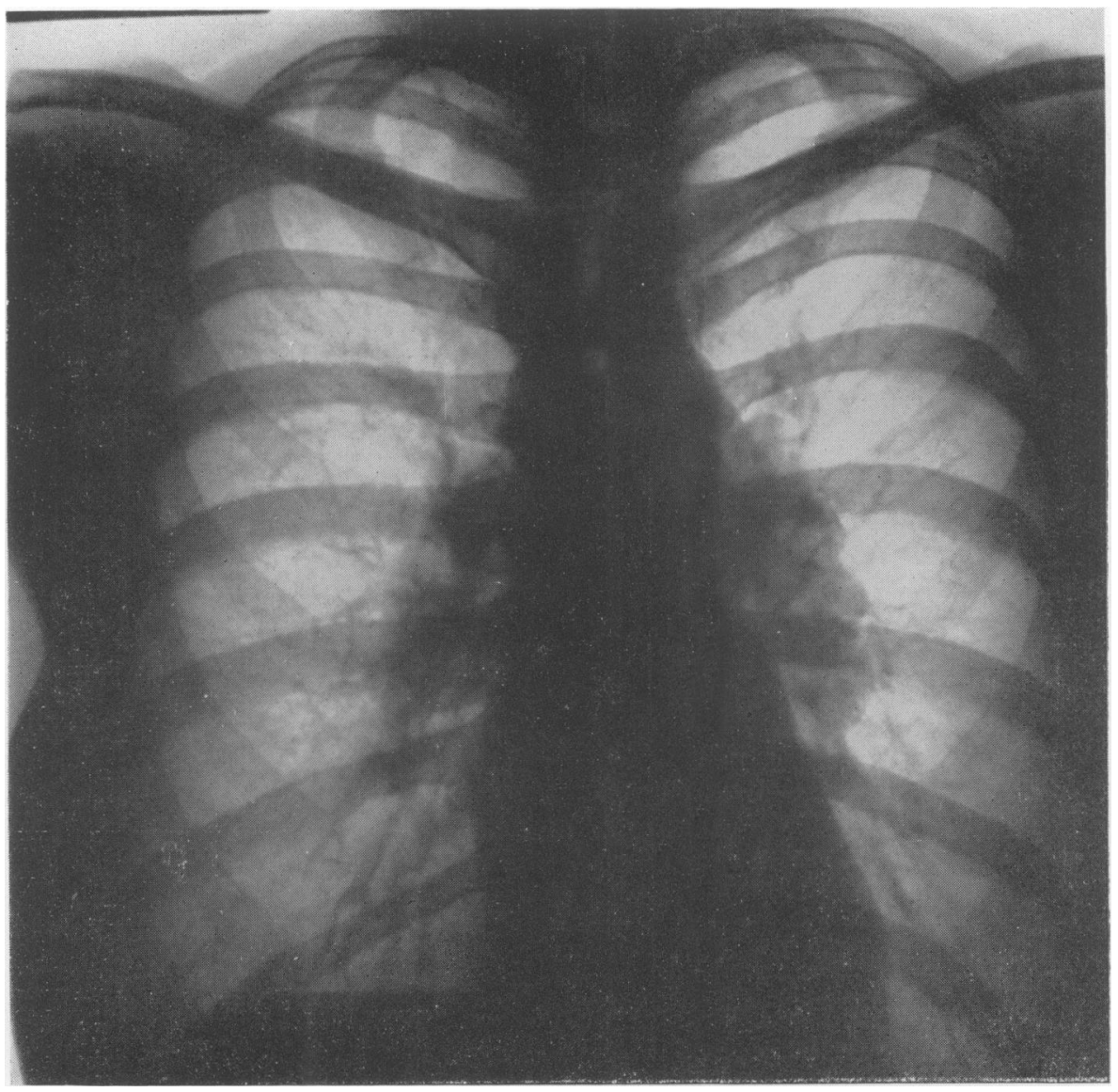

FIG. 3.-X-ray showing the characteristic bilateral tuberous enlargement of the hilar shadows. The lung fields are clear.

In assessing the progress of lung disease, symptoms are of little value as they are present to a significant degree only in the advanced fibrotic cases or in those developing complications such as caseating tuberculosis or right heart failure. We depend almost entirely on the X-ray appearances. The case illustrated in Figs. 5 and 6 gives an unusual opportunity for relating the disease, present in the lung in a comparatively early stage, to that seen in the X-ray. There is a remarkable difference in the relative densities, though this is exaggerated as the lung biopsy is not inflated. The common fallacies of X-ray interpretation are well known, though they are sometimes conveniently forgotten. It is commonly accepted that a clearing of the picture is, in the absence of symptoms, a return of the lung to a normal state. While this is a practical, and probably correct belief, we must remember that there are at present few physiological long-term studies of these recovered cyses. The lungs illustrated in Fig. 4 cleared after the in- filtrations had persisted for one year. Six month after a normal picture was obtained, a punc. biopsy of a normal sized liver showed activi sarcoid lesions.

In most cases of sarcoidosis there are no factores which allow prognostic grouping. There arę however, two types of the disease which can reasonably be separated on this account. Beryllium is responsible for a condition so similar in mant. respects to other forms of sarcoidosis that we mañ speak of beryllium sarcoid. This is discusse further below, but one of its distinctive features the rarity of resolution and consequent poor progf nosis. A second group of cases present with erythema nodosum. These cases, in contrao distinction to the former group, have an excellert outlook and clearing of the infiltrations is the rule.

All aspects of pulmonary sarcoidosis are con sidered in detail by Scadding in the Bradshaw lecture for 1949 (Scadding, 1950). 


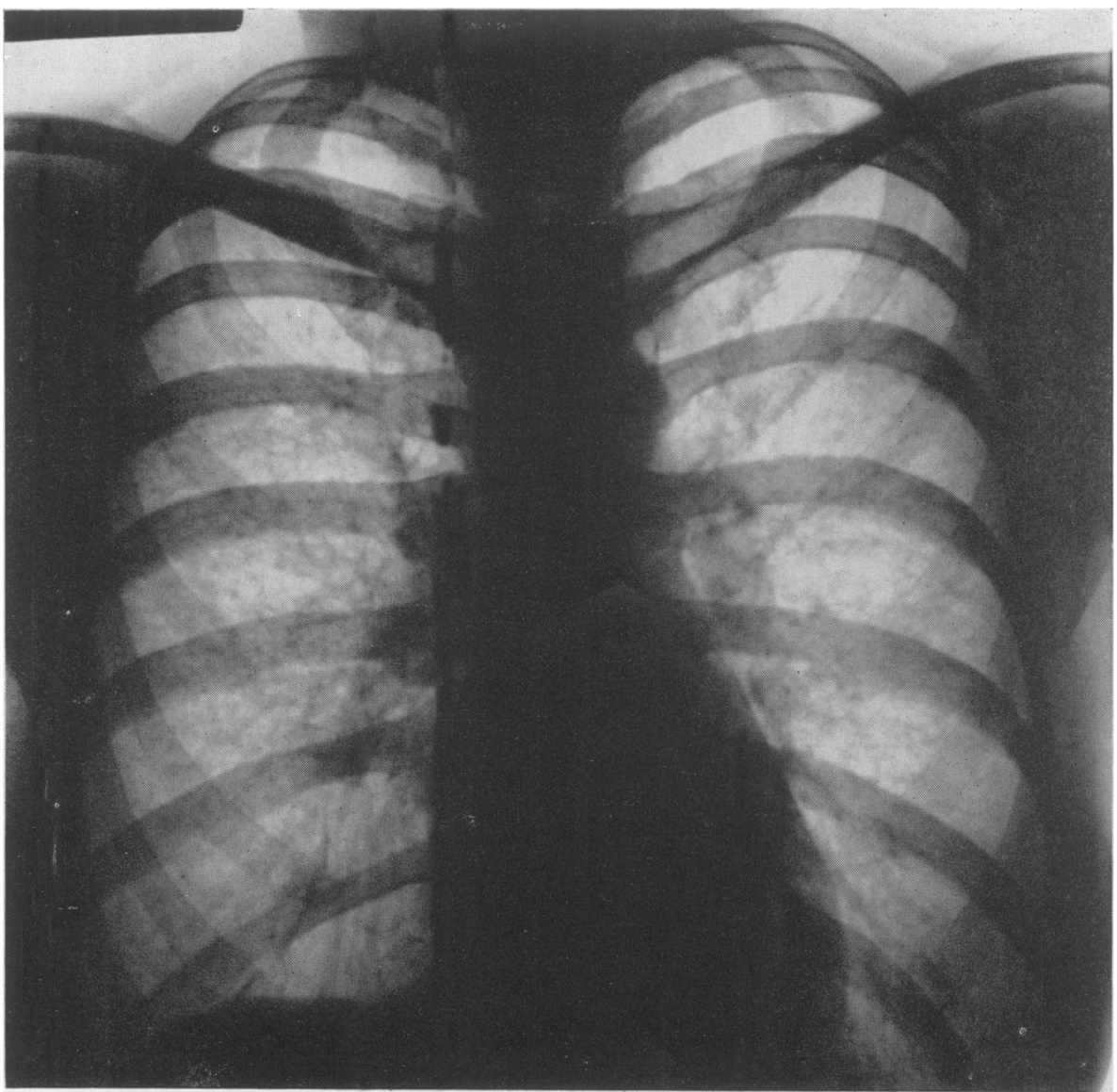

Fig. 4.-Same case as Fig. 3, nine months later. The hilar shadows are almost normal. There is scattered medium-sized mottling throughout both lungs.

\section{The Skin}

It is usual in discussing the skin changes associated with sarcoidosis to consider them in three groups. The first and perhaps the commonest is Boeck's ' multiple benign sarkoid,' or as he termed it later ' miliary lupoid.' Secondly there is the subcutaneous sarcoid of Darier and Roussy (1904), and lestly lupus pernio, first described by Besnier in 1889 . The first two groups may be associated with each other, but lupus pernio generally appears on its own. Multiple benign sarcoid is formed by nodules or plaques. The lesions are at first reddish or bluish-red, darkening to yellowish-brown. On pressure they show small gr eyish-yellow foci from which appearance derives the name miliary lupoid. They are very variable in number, size and distribution, being most commonly seen on the face, back or trunk and extensor aspects of the limbs. The lesions may persist unchanged or resolve with scar formation.
Ulceration does not occur. The subcutaneous sarcoid lesions of Darier-Roussy are much less frequent. The deposits are more deeply-seated, comparatively few in number, of normal skin colour or dull bluish-red, and in size as big as a walnut. They may be accompanied by the lesions of the Boeck type.

Lupus pernio is distinct from the preceding types and appears as a rule on its own. It is characterized by symmetrical erythematous areas with bluish-red infiltrated thickened plaques. Telangiectases may be prominent and ulceration is common. It affects especially the nose, face, ears, fingers and back of hands. In old lesions tissue loss with atrophy, ulceration and scarring is noticeable.

\section{The Eye}

Iritis is the most frequent and important of all ocular sarçoid lesions. Characteristically it is a 


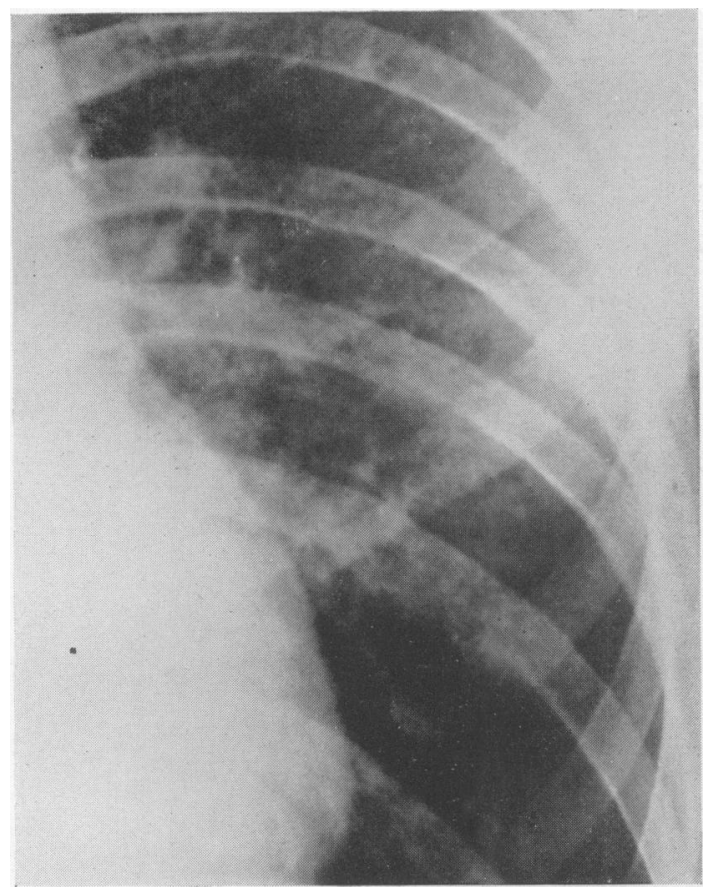

FIG. 5--X-ray of left mid-zone showing sarcoid infiltration, part of which is in the apex of left lower lobe.

painless nodular iritis with only slight inflammatory symptoms (Woods, I95I). The nodules are larger, pinker and more irregular than miliary tubercles. Large 'mutton fat' keratic deposits are common. 'The disease is chronic, with remissions, and may heal with hyalinization or complete regression. Unfortunately there is a serious risk of phthisis bulbi with complete blindness.

Occasionally the eye lesions are accompanied by enlargement of the lacrimal, salivary and cetvical lymph glands. Such cases have been reported as uveo-parotitis and in some instances Mikulicz syndrome.

\section{Bones}

Holt and Owens (1949) in their excellent survey of skeletal sarcoidosis say that a reticular pattern resulting from the destruction of the finer trabeculae is the most characteristic and commonest $\mathrm{X}$-ray finding. It is seen especially in the phalanges and is almost specific for sarcoidosis. This change may be accompanied by the often-mentioned 'punched out' cystic spaces (Figs. 7 and 8). The disease in the bone starts in the medullary cavity and the earliest change noted is a mottled rarefaction or stippled pattern in the phalanges. This tends to be more marked at the distal ends of the proximal and middle phalanges and the proximal ends of the distal phalanges, though any part of the skeleton may be involved. The्ष्त lacunae formed enlarge and coalesce to form the classical cysts in various shapes and sizes. Hol and Owens' paper well illustrates these changes? They estimate the incidence of bone changes at: around 15 per cent., but this is a rough figure and much depends on the thoroughness with whicto the lesions are sought. They also reaffirnof Schaumann's original observation (Schaumannow 1924) that extensive sarcoid infiltration of the bone marrow may be present without any clinicad or radiological evidence of disease. Finger swell $\stackrel{\text { s }}{\circ}$ ings, when present, are generally painless. The bone disease may heal with resolution or fibrotie persistence of the 'cysts.' Occasionally spon taneous fractures and gross finger deformity mas ensue.

\section{The Nerious System}

Colover (1948) has reviewed the changes that are sometimes found in the nervous system. Thie cranial nerves are most often involved, especiallo the seventh on one or both sides, sometimes accompanied by parotid enlargement. The optic nerve and the glosso-pharyngeal and vagus, wits affection of the pharynx and vocal cords, are alse. liable to infiltration. The peripheral nerves mar be affected. Meningo-encephalitis and myegtis have been seen.

\section{The Heart}

Sarcoid infiltration of the myocardium is not common. Most of the cases reported have beeñ in the American literature, and like the disease itself in that country, most instances have been $i \vec{p}$ negroes. Clinically it may be suspected in the presence of various arrythmias or unexplained tachycardia. It has been responsible for sever cases of sudden death in apparently healtho people (Simkins, I95 I).

\section{Other Organs}

It is common for the liver to show foci even when it is of normal size and there is, as a rule, no detectable impairment of function. The splees shares the involvement of other lymphatic struo tures, is often palpable and may rarely be ver large. The kidneys are seldom clinically recogo nized as being affected. Many other parts of the body on occasions may be diseased, including the nose, larynx, tonsils and adenoids, epididymis; testes and breasts. The pituitary and thyroe have also shown infiltration.

\section{Criteria of Diagnosis}

The factors necessary before making the diage nosis of sarcoidosis in any case will depend on the standards and beliefs of the observer, and tree purpose for which the diagnosis is required. 


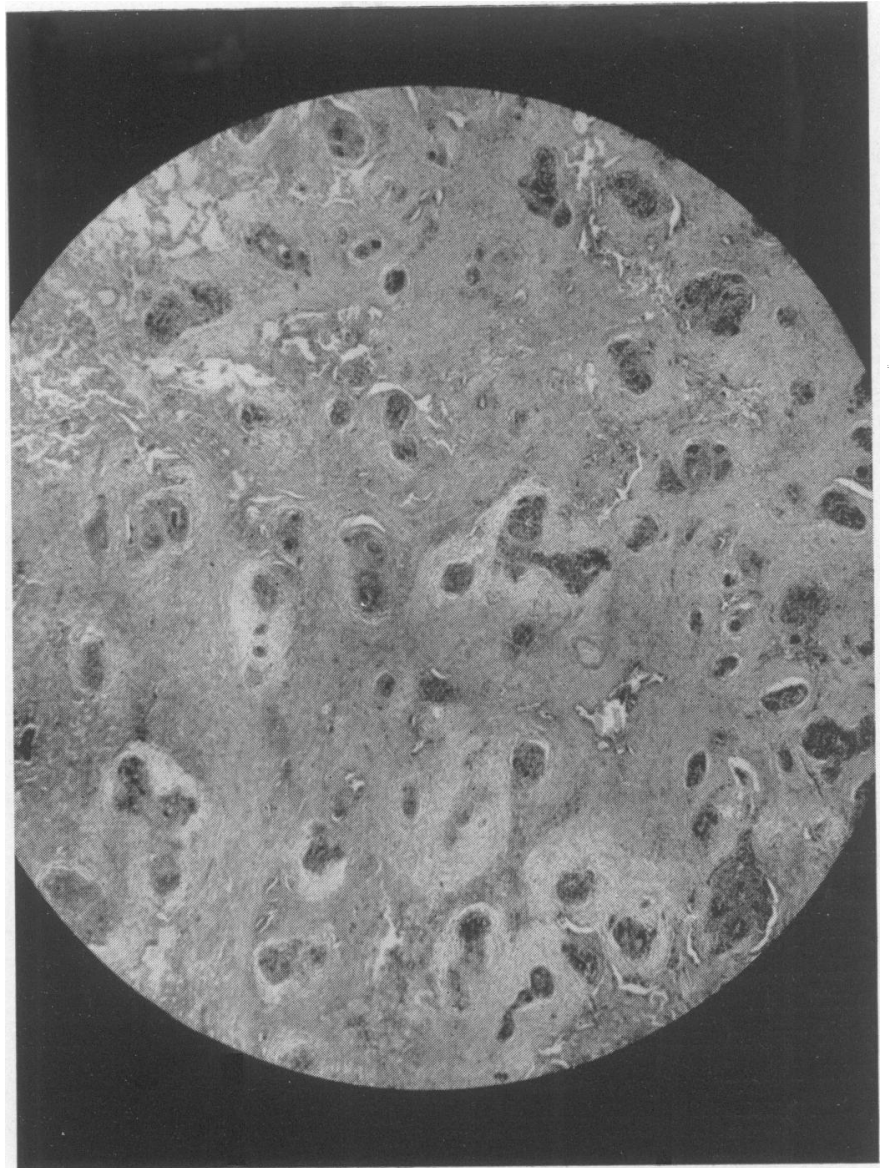

Fig. 6.-Same case as Fig. 5. Section from biopsy of apex of left lower lobe showing numerous sarcoid foci. For comparison with X-ray.

In all cases it is essential that there are present some of the accepted proven clinical features. A single bizarre lesion, even with the standard histological appearances, is not adequate. On the other hand, in clinical practice, it may be sufficient to note the typical X-ray picture of the lungs and mediastinum together with the state of the patient, to make a sufficiently assured diagnosis for the well-being and treatment of the patient. However, most clinicians would agree that, especially in the present state of our knowledge of this disease, wherever possible biopsy evidence should support the clinical picture. This has been found most often in the superficial lymph glands, even where they are hardly enlarged. The skin, the liver, the tonsil, the nasal mucosa and sternal marrow have also been found convenient sites. The case illustrated in Fig. 2 was diagnosed ky biopsy of a mediastinal gland removed at the time of ligature of ductus arteriosus. As similar changes are found in the mediastinal glands in pulmonary tuberculosis, the diagnosis was only accepted after further clinical development. The lung is also a rare source of biopsy material (Fig. 6).

\section{Aetiology}

The agent responsible for the development of sarcoidosis is unknown. In England it is generally held that most cases are a result of a peculiar reaction to the tubercle bacillus. This view has many antagonists, especially on the Continent.

The high incidence of tuberculin anergy, the finding of tubercle bacilli, sometimes only after prolonged search, in cases fulfilling other criteria of sarcoidosis are evidence used to support the tuberculous aetiology. Kyrle's inoculation experiments with tubercle bacilli (Kyrle, I92I), the papules and glands developed by B.C.G. in sarcoid patients, and the changes found in the glands 


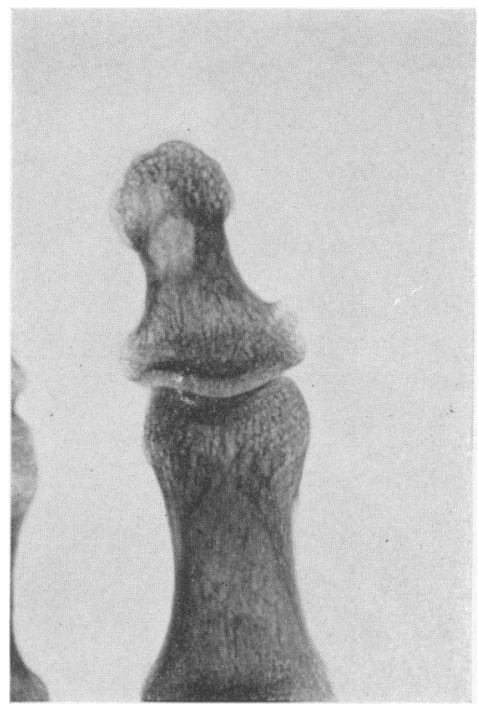

FIG. 7.-X-ray of big toe showing 'cyst' in terminal phalanx.

draining caseous tuberculous lesions are facts which show that the tubercle bacillus can produce the 'sarcoid' histological picture without the bacillus being demonstrable and without caseation. But perhaps the most convincing evidence is the clinical experience that all gradations from open pulmonary tuberculosis on the one hand, to classical sarcoidosis on the other, are not infrequently met.

None of this evidence is absolute and all has been refuted to the satisfaction of the opponents of the tuberculous basis of sarcoidosis. The rarity of sarcoid infiltration of the bronchi, pleura joints and adrenals, in spite of its wide dissemination, are also notable and perhaps unexpected differences.

Some authorities hold that the disease is due to a specific but unknown virus. Kveim's test, discussed earlier, is claimed to support this view. Others believe that various fungi are responsible.

The chronic granuloma associated with beryllium poisoning is very similar to sarcoidosis, though Hardy (195I) believes that the two conditions can be separated both by their histological appearances and by their clinical behaviour. The chronic beryllium disease is characterized by severe respiratory disability early on, and by the prevalence of gastro-intestinal symptoms. It has a bad prognosis.

A dual aetiology has been suggested. The work of Kallos (Warfinge, 1943) lends support to this idea. White rats are endemically infected with bartonella. If they are inoculated with virulent tubercle bacilli their tissues respond in a sarcoid

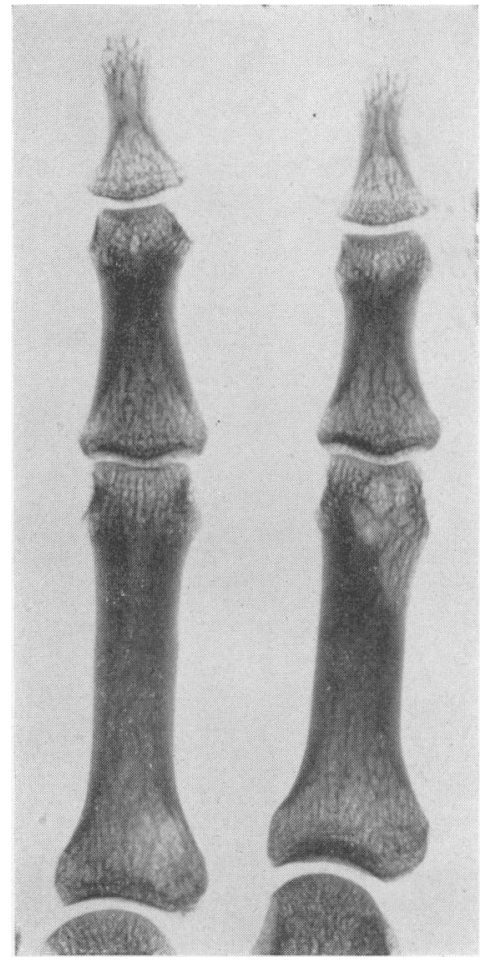

FIG. 8.-X-ray showing cystic sarcoid changes in distal end of proximal phalanx of index finger. There are also less obvious changes in proximal end of same phalanx in middle finger.

manner. If, however, the rats' bartonella infection is countered effectively and their reticulo-endo응 thelial system blocked with indian ink, then the tubercle bacilli produce caseating tuberculosis.

The group of cases presenting with erythema nodosum is also worth commenting on in this con 3 . nection. This type of sarcoidosis is almost con fined to women of child-bearing age. After the initial febrile reaction, with the typical and non 8 specific rash, the course is that of pulmonary sarcoidosis with an excellent prognosis. It is widely held that erythema nodosum is a $\operatorname{sen}_{3}$ sitivity response to a variety of agents, the tubercle bacillus, the streptococcus, sulphathiazole, etc. If the tubercle bacillus is responsible for the eryos thema group it is difficult to understand how the body can react with low skin sensitivity to tubercu代 lin at the same time as the erythema nodosum is iv evidence. We can postulate that some othes product of the tubercle bacillus is causing the erythema nodosum or, as is more likely, some other substance is present, perhaps from a strepto=coccus, which is not only responsible for the erythema, but also modifies the body's reaction to the tubercle bacillus in this very peculiar way. IS 
is all unproven conjecture. At present we may conclude that there are several agents responsible, and as Scadding suggests (I950) we may speak of tuberculous sarcoidosis, of beryllium sarcoidosis, or of sarcoidosis due to any other agent that may possibly in the future be shown to cause it.

\section{Treatment}

In assessing the effectiveness of any particular line of therapy we depend on observations of localized disease, especially in the lungs, skin or eyes. The difficulties are at once apparent. The progress of the local lesion is not necessarily a true indicator of the state of the generalized disease. It is a frequent observation that decrease in the size of the hilar glands is succeeded by lung dissemination. Uveitis may heal only to be followed years later by other overt lesions in the skin, lungs or elsewhere. The unpredictable variations in the natural course of the local disease have been mentioned above. The whole disease may, with evanescent'symptoms, apparently resolve in a year, for example the erythema nodosum group. In contrast the beryllium cases progress relentlessly with little relief. One of Boeck's original cases dying of carcinoma 29 years after the original diagnosis well illustrates the extreme chronicity of some cases (Danbolt, 1936). At autopsy the scars of the initial lesions on the cheeks were accompanied by more recent active skin disease, and similar foci were found in bones, lymphatic glands, lungs and spleen.

With such variety of behaviour before us demanding caution, we can more reasonably appreciate the value of any therapeutic measure against the whole disease process. At the same time we should remember that death usually results from lung involvement and distress from blindness and skin deformity. If treatment is effective in these sites alone it is still of great benefit to the patient, although it is true that the local lesion may not reflect the thera- peutic response of the disease as a whole.

Most workers have treated a few cases with streptomycin and P.A.S. in similar dosage to that used for pulmonary tuberculosis. In general their results suggest that this treatment has little or no effect on the course of the disease.

Calciferol has given much more encouraging results. Scadding (1950) reports its use in pulmonary disease. He gave 50,000 to I 50,000 units daily, depending on the tolerance of the patient. He concluded that it was well worth further investigation. It cannot be expected to help in the long-standing fibrotic lesions, but infiltrations of several years' persistence may clear, and in doubt there is little to lose by careful trial. Favourable results are also reported in treatment of the skin lesions.

Recently cases have beèn treated by cortisone and A.C.T.H. (Siltzbach et al., $195 \mathrm{I}$; Lovelock et al., I95I ; Small, 195I). It is much too early to determine the true value of these measures. There is often prompt and encouraging response with rapid decrease in the size of liver and spleen, and a general sense of well being. If respiratory disability was present it may show improvement. Unfortunately, the duration of the benefit in some cases is disappointing, relapse occurring a few weeks after the cessation of treatment. The results may well depend on the age of the lesion and the causative agent.

I have reviewed the main features of sarcoidosis and have considered its diagnosis, aetiology and treatment. There is still much that is uncertain and unknown about this fascinating group of diseases. In advancing our knowledge and increasing our experience of this condition we should avoid, on the one hand, a too rigid limitation of criteria, and on the other we should hesitate to include borderline cases unsupported by sufficient evidence.

I am indelted to Dr. J. G. Scadding for the frontispiece and for Figs. 7 and 8, and to Dr. J. Clegg for Figs. 5 and 6.

\section{BIBLIOGRAPHY}

BESNIER, E. (1889), Ann. Dermat. Syph., 10, 333.

BOECK, C. (1899), F. Cutan. G.U. Dis., 17, 543 .

COLOVER, J. (1948), Brain, 71, $45 \mathrm{I}$.

DANBOLT, N., and HVAL, E. (1936), Acta. Derm. Venereol., 17, 477.

DANBOLT, N. (I95I), Ibid., 31, 184 .

DARIER J., and ROUSSY, G. (190\%), Ann. Dermat. Syph., 5, 144. FREIMAN, D. G. (1948), New Eng. F. Med., 239, 664, 709, 743. HARDY, H. L. (I95I), Proc. R. Soc. Med., 44, 257.

HOLT, J. F., and OWENS, W. I. (1949), Radiology, 53, I. - -

HUNTER, F. T. (1936), New Eng. F. Med., 214, 346.

HUTCHINSON, J. (1878), 'Illustrations of Clinical Surgery,' p. 42.

HUTCHINSON, J. (1898), Arch. Surg., 9, 307.

ISRAEL, H. L., SONES, M., STEIN, S. C., ARONSEN, J. D. (195C), Amer. Rev. Tuberc., 62, 408.

KVEIM, A. (i94I), Nord. Med., 9, 169.

KYRLE, T. (r92 1), Arch.f. Dermat. Syph., 131, 33.
LEMMING, R. (1940), Acta. Med. Scand., 103, 400.

LEMMING, R. (1942), Ibid., I10, 151 .

LOVELOCK, F. J., and STONE, D. J. (r951), f. Amer. med. Ass., I47, 930 .

NELSÓN, C. T. (1949), Arch. Derm. Syph., 60, 377.

RICKER, W., and CLARK, M. (1949), Amer. F. Clin. Path., 19, RUBIN, E. H., and PINNER, M. (1944), Amer. Rev. Tuberc., 49,

SCADDING, J. G. (1950), Brit. Med. F., i, 745.

SCHAUMANN, J. (1924), Brit. F. Derm. Syph., 36, 515.

SILTZBACH, L. E., POSNER, A., MEDINE, M. M. (I951), F. Amer. med. Ass., 147, 927.

SIMKINS, S. (195I), Ibid., 146, 794.

SMALL, M. J. (1951), Ibid, 147. 932.

WARFINGE, L. E. (1943), Acta. Med. Scand., 114, 259.

WELLS, A. Q., and WYLIE, J. A. H. (I949), Lancet, i, 439.

- WOODS, A. C. (1949), Trans. Amer. Acad. Ophthal. Otolaryng., 